\title{
Neurorretinitis por Bartonella henselae en un adolescente
} Bartonella henselae neuroretinitis in an adolescent

\author{
Dra. María E. Conde ${ }^{a}$ Dra. María E. Antueno ${ }^{a}$ Dra. Florencia Palmieri ${ }^{a}$ Dra. María V. López $z^{a}$ \\ Dr. Ariel Cheistwera
}

\begin{abstract}
RESUMEN
La neurorretinitis como manifestación de la enfermedad por arañazo de gato se presenta en el 1-2 \% de los pacientes con afecciones oculares por Bartonella henselae. Las manifestaciones oculares suelen suceder a las sistémicas, aunque pueden aparecer en ausencia de estas. La presencia de exudado macular en forma de estrella es característico y sugestivo de dicha infección.

Se presenta el caso de un paciente de 14 años de edad, previamente sano, con disminución de la agudeza visual de 15 días de evolución, queingresó por sospecha de neuritis óptica izquierda con edema de papila. El seguimiento oftalmológico reveló la aparición de la lesión característica en "estrella macular" que permitió arribar al diagnóstico de infección por B. henselae, confirmándose luego con serología positiva. Palabras clave: Bartonella henselae, neurorretinitis, papilitis óptica, estrella macular.
\end{abstract}

\section{ABSTRACT}

Neurorretinitis as a manifestation of cat scratch disease occurs in 1-2\% of patients with Bartonella Henselae eye disease. Ocular manifestations tend to follow systemic ones, although they can appear in their absence. The presence of star-shaped macular exudate is characteristic and suggestive of this infection.

We report a case of a 14-year-old healthy boy, with 15 days of decreased visual acuity, who was admitted for suspected left optic neuritis with papilledema. Ophthalmological examination revealed the characteristic "macular star" that led to the diagnosis of infection by Bartonella Henselae, later confirmed by positive serology.

Key words: Bartonella Henselae, neuroretinitis, optic papillitis, macular star.

http:/ / dx.doi.org/10.5546/ aap.2021.e616

Cómo citar: Conde ME, Antueno ME, Palmieri F, López MV, Cheistwer A. Neurorretinitis por Bartonella henselae en un adolescente. Arch Argent Pediatr 2021;119(6):e616-e620.

a. Unidad 6 de Clínica Pediátrica, Hospital de Niños Ricardo Gutiérrez, Ciudad Autónoma de Buenos Aires, Argentina.

Colaborador:

Dr. Mario Villarrubia. Hospital de Niños Ricardo Gutiérrez.

Correspondencia:

Dra. Florencia Palmieri: flor.palmieri@gmail.com

Financiamiento: Ninguno.

Conflicto de intereses: Ninguno que declarar.

Recibido: 5-11-2020

Aceptado: 10-6-2021

\section{INTRODUCCIÓN}

La enfermedad por arañazo de gato es causada por un bacilo gramnegativo, Bartonella henselae, y suele ser una infección autolimitada. Se transmite por mordedura, lamida o rasguño de un animal infectado, a menudo gatos y perros, pero también otros menos frecuentes como las ardillas y las cabras, y por las pinzas de los cangrejos..$^{1-3} \mathrm{La}$ infección primaria puede presentarse como una mácula, una pápula no pruriginosa o una vesícula en el sitio de inoculación que 3 a 5 días después se acompaña de síntomas gripales y linfadenopatía regional. ${ }^{1,2}$ En raras ocasiones, puede producirse diseminación hematógena que da lugar a complicaciones sistémicas más graves, como abscesos esplénicos, hepatitis granulomatosa, neumonía, osteomielitis, afectación ocular y neurológica. ${ }^{2}$

Después de la linfadenopatía regional, la afección ocular es la manifestación sistémica más común de la enfermedad, exceptuando los órganos linfáticos. Dentro de esta, el síndrome oculoglandular de Parinaud, caracterizado por infección de la conjuntiva y los párpados, asociado con linfadenopatía regional, ${ }^{4}$ junto a los infiltrados retinianos, son las presentaciones más frecuentes. La neurorretinitis (edema de disco óptico acompañado de estrella macular) solo ocurre en el 1 a $2 \%$ de los casos de infección sistémica por B. henselae. ${ }^{1}$ A pesar de ello, la infección por dicho microorganismo debe ser considerada en pacientes con afección ocular de causa desconocida. ${ }^{5}$

\section{CASO CLÍNICO}

Paciente de sexo masculino de 14 años de edad, sin antecedentes personales de relevancia, consultó en otro centro por visión borrosa y disminución súbita de la agudeza visual del ojo izquierdo de 15 días de evolución. No refirió otros síntomas como vómitos o cefalea, ni antecedente de traumatismo. Durante la anamnesis surgió el contacto con gatos días previos al episodio. Por sospecha de neuritis óptica izquierda, fue derivado a nuestro hospital para realizar diagnóstico y tratamiento. 
$\mathrm{Al}$ ingreso, se lo evaluó en buen estado general. El examen neurológico se encontraba dentro de límites normales, las pupilas isocóricas y reactivas, el reflejo fotomotor directo y consensual conservados, el reflejo corneal presente y la motilidad ocular extrínseca conservada.

Se le realizó evaluación oftalmológica en donde se observó agudeza visual disminuida en ojo izquierdo de 3/10 y normal en ojo derecho, con papilitis y edema del disco óptico evidenciado por tomografía de coherencia óptica (OCT, por su sigla en inglés) del ojo afectado (Figuras 1 y 2).
Durante la internación se realizaron diversos exámenes complementarios para descartar patología desmielinizante, entre ellos resonancia magnética nuclear (RMN) de órbita, sistema nervioso central (SNC) y columna sin hallazgos patológicos, y potenciales evocados visuales que informaron latencia prolongada en ojo derecho y al estímulo de ojo izquierdo, con electrorretinograma normal, sugiriendo anormalidad prequiasmática a predominio izquierdo. Se desestimó compromiso del lado derecho por presentar agudeza visual 10/10 y

FIGURA 1. Tomografía de coherencia óptica papilar

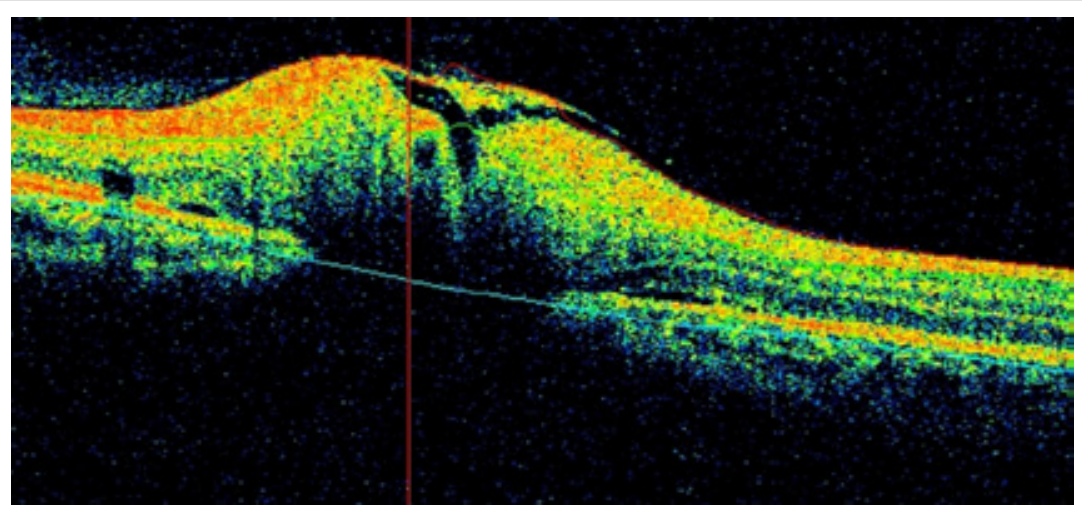

Se observa edema de papila franco.

FIGURA 2. Tomografía de coherencia óptica de capa de fibras nerviosas de la retina

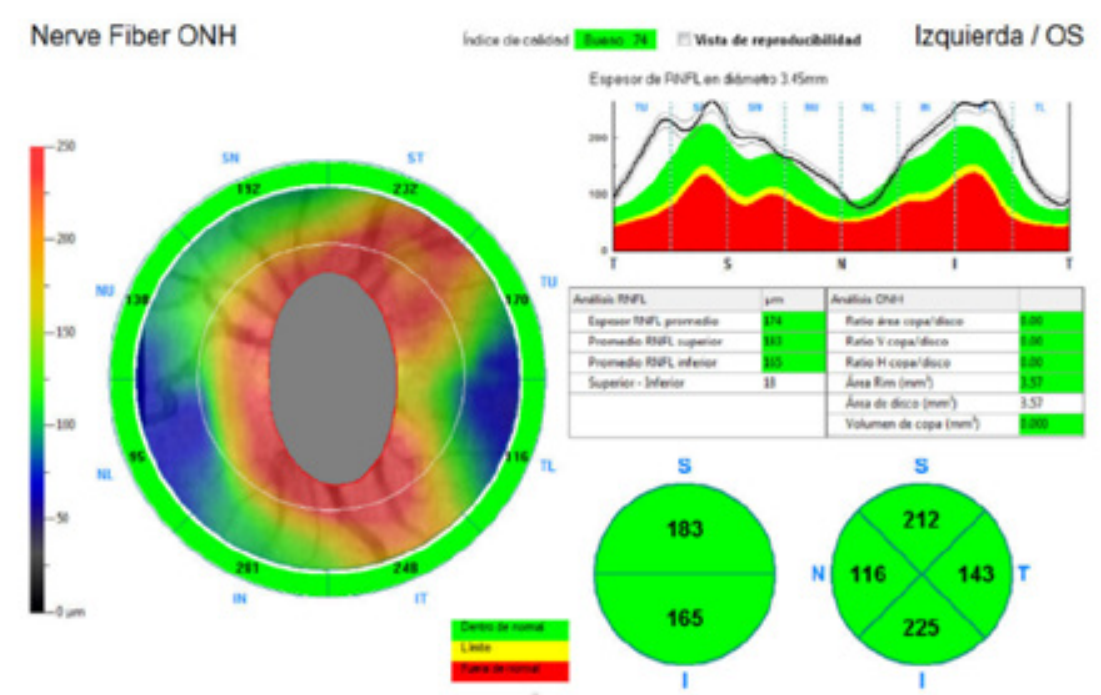

Se observa el aumento del espesor de la capa de fibras peripapilares (valores normales por debajo de 140), que muestra con valores objetivos el edema de esta capa, en el rango de papiledema.

OCT: tomografía de coherencia óptica (por su sigla en inglés). ONH: cabeza del nervio óptico (por su sigla en inglés). RNFL: capa de fibras nerviosas (por su sigla en inglés). SN: superior nasal. ST: superior temporal. IN: inferior nasal.

IT: inferior temporal. NL: nasal bajo (por su sigla en inglés). TL: temporal bajo (por su sigla en inglés).

NU: nasal alto (por su sigla en inglés). TU: temporal alto por su sigla en inglés). 
estudios por imágenes normales.

Al tercer día de internación se realizó nueva evaluación oftalmológica en la que se evidenciaron papilitis y edema macular leve, agregándose una lesión en estrella macular en el ojo afectado (Figura 3). Se realizó una nueva OCT (Figura 4) que mostró exudados lipídicos duros en las capas externas de la retina con mejoría del edema papilar respecto a días previos, continuando con el compromiso de la agudeza visual (ojo izquierdo: 2/10).
Se realizaron los siguientes estudios de laboratorio: hemograma, función renal, hepatograma, glucemia y reactantes de fase aguda, todos ellos dentro de límites normales. Se solicitaron serologías para citomegalovirus (CMV), virus de Epstein Barr (EBV), Toxoplasma, Toxocara y sífilis (RPR), todos con resultados negativos. Se obtuvo retrospectivamente, luego de 2 semanas, resultado positivo para inmunoglobulina M (IgM) e inmunoglobulina $G$ (IgG), (títulos 1/64) para B. henselae.
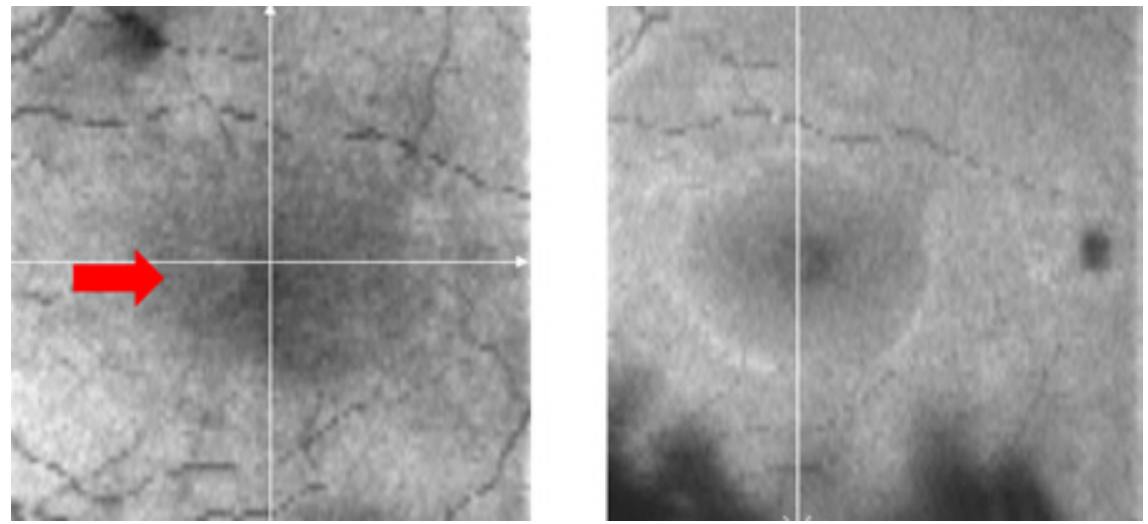

En la imagen de la izquierda, se puede apreciar una banda hipopigmentada en región foveolar (flecha roja) correspondiente a una parte de los exudados duros dispuestos radialmente (estrella macular). En la imagen de la derecha, se observa su reabsorción al finalizar el tratamiento.

FIGURA 4. Tomografía de coherencia óptica macular de diferentes alturas del ojo izquierdo
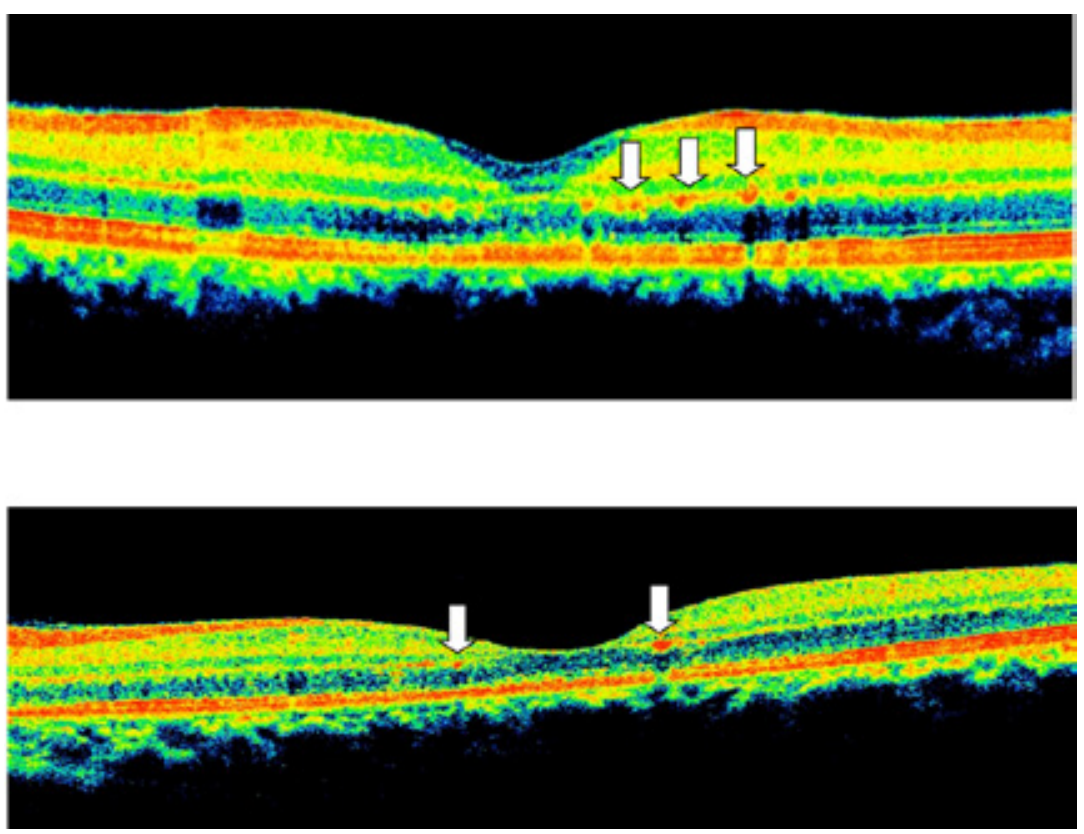

Se pueden apreciar los exudados lipídicos duros en capas externas de la retina señalados con flechas. 
Inicialmente, con diagnóstico presuntivo de neuritis óptica izquierda, se indicaron tres pulsos de corticoides con escasa respuesta.

Luego de la realización del fondo de ojo a las 72 horas de internación, con el hallazgo de la estrella macular sugestiva de infección por $B$. henselae, se inició tratamiento antibiótico con trimetoprima sulfametoxazol (TMS) durante 5 días. Luego se cambió a doxiciclina, el antibiótico más comúnmente utilizado para esta patología. Se confirmó el diagnóstico retrospectivo con las pruebas serológicas positivas para $B$. henselae.

El paciente permaneció internado durante 13 días, evolucionando con leve mejoría de la agudeza visual. Recibió 15 días totales de meprednisona, luego de los tres pulsos con metilprednisolona, y cumplió 60 días totales de tratamiento antibiótico con doxiciclina, tiempo mayor a la duración del tratamiento habitual por haber realizado un seguimiento pediátrico errático. Concurrió luego de 3 meses a control al Servicio de Oftalmología, donde se evaluó agudeza visual de ojo izquierdo 9/10 con franca mejoría en relación con la última consulta, OCT con perfil macular conservado y fondo de ojo homolateral con estrella macular remanente.

\section{DISCUSIÓN}

La enfermedad por arañazo de gato es una infección sistémica causada por el bacilo gramnegativo $B$. henselae. La manifestación principal es una linfadenitis subaguda en el área de inoculación. Si bien la forma diseminada de la enfermedad es rara, el espectro puede variar desde una simple linfadenitis a una endocarditis, compromiso óseo cuyos sitios más comúnmente afectados son la columna vertebral y la cintura pélvica, o la afección hepatoesplénica. En la forma diseminada, el ojo es el órgano no linfático más comúnmente afectado, y ocurre entre el $5 \%$ y el $10 \%$ de los pacientes. ${ }^{3}$

Existen múltiples formas de presentación ocular, entre ellas se destacan la pérdida de la agudeza visual generalmente unilateral, el defecto aferente, la discromatopsia y los defectos del campo visual. El signo más clásico es la neurorretinitis, que se caracteriza por la pérdida de la agudeza visual abrupta y de progresión rápida, síntomas coincidentes con el inicio del cuadro del paciente mencionado en este apartado. ${ }^{6}$

En la práctica clínica, se evidencia en el fondo de ojo una estrella macular característica. Suele presentarse varios días después de la pérdida de agudeza visual, generalmente luego de 2 a 3 semanas, como ocurrió en el caso de este paciente, en donde no se evidenció sino hasta 3 semanas posteriores al inicio de los síntomas. ${ }^{7}$

En lo que respecta a los estudios por imágenes, la tomografía de coherencia óptica ha sido utilizada para detectar hallazgos retinianos que no son obvios en el examen clínico. Los exudados retinianos en la capa plexiforme externa correspondientes a la estrella macular pueden observarse antes de que sean directamente visibles para el ojo del examinador. ${ }^{8}$

Debido a que la formación de la estrella macular puede no evidenciarse al inicio del cuadro, el diagnóstico serológico de infección por $B$. henselae cobra vital importancia en pacientes con compromiso ocular y sospecha de neurorretinitis, como ocurrió con el paciente presentado.

Las pruebas serológicas basadas en la detección de inmunoglobulina G ( $\operatorname{IgG}$ ) e inmunoglobulina M (IgM) son confiables. La IgM positiva indica enfermedad aguda. Los títulos de IgG superiores a 1:256 confirman la enfermedad. Los títulos entre 1:64 y 1:256 sugieren una posible enfermedad por arañazo de gato, y la serología debe repetirse 10-14 días después. En el caso presentado, no se consideró necesario repetirla debido a la sumatoria de hallazgos oftalmológicos y serológicos. ${ }^{4}$

La reacción en cadena de la polimerasa (PCR, por su sigla en inglés) es una técnica más avanzada que se ha utilizado recientemente para el diagnóstico, pero existen pocos informes sobre la PCR en humor acuoso. ${ }^{4}$

En lo que respecta al tratamiento, el uso de antibióticos y corticoides es controversial y varía según la bibliografía, debido a la tendencia de esta infección hacia la resolución espontánea.

Algunos autores hacen referencia a que no existe evidencia de mejoría con el uso de corticoides, ${ }^{9-11}$ aunque se plantea la posibilidad de que sus efectos antiinflamatorios aporten algún tipo de beneficio en casos graves. ${ }^{11}$

En lo que respecta al tratamiento antibiótico, hay autores que afirman que solo estaría indicado en pacientes inmunocomprometidos. ${ }^{1}$ Otros también sugieren tratar a los pacientes inmunocompetentes con síntomas sistémicos moderados a graves o pérdida significativa de la visión, como sucedió en el paciente citado. ${ }^{11}$ Entre los antibióticos más utilizados se citan: doxiciclina, ciprofloxacina, claritromicina, azitromicina, rifampicina y trimetoprima- 
sulfametoxazol. Algunos mencionan la terapia combinada (principalmente doxiciclina o azitromicina-rifampicina), durante 4 a 6 semanas, plazo que puede extenderse en pacientes inmunocomprometidos. ${ }^{3,7,11-13}$

En síntesis, si bien la neurorretinitis aislada como manifestación de la infección por B. henselae es infrecuente, es necesario considerar dicha etiología ante la disminución aguda de la agudeza visual, y especialmente frente al hallazgo de papiledema y estrella macular en el fondo de ojo, teniendo en cuenta que esta última puede no estar presente al inicio de los síntomas oculares.

\section{REFERENCIAS}

1. Abazari A, Kaplowitz K, Sibony P. A 31-year-old man with bilateral blurry vision and floaters. DigitJ Ophthalmol.2015; 21(2):1-11.

2. Solley WA, Martin DF, Newman NJ, King R, etal.Cat scratch disease: posterior segment manifestations. Ophthalmology. 1999; 106(8):1546-53.

3. Ghazi NG, Sams WA. A case of cat-scratch disease with unusual ophthalmic manifestations. Middle East Afr J Ophthalmol. 2012; 19(2):243-6.
4. Ksiaa I, Abroug N, Mahmoud A, Zina S, et al. Update on Bartonella neuroretinitis. J Curr Ophthalmol. 2019; 31(3):254-61.

5. Golnik KC, Marotto ME, Fanous MM, Heitter D, et al. Ophthalmic manifestations of Rochalimaea species. Am J Ophthalmol. 1994; 118(2):145-51.

6. Saatci OA, Oner FH, Kargi A, Kavukcu S. Unilateral neuroretinitis and periparillary serous retinal detachment in cat-scratch disease. Korean J Ophthalmol. 2002;16(1):43-6.

7. Oray M, Önal S, Koç Akbay A, Tuğal Tutkun İ. Diverse Clinical Signs of Ocular Involvement in Cat Scratch Disease. Turk J Ophthalmol. 2017; 47(1):9-17.

8. Abdelhakim A, Rasool N. Neuroretinitis: a review. Curr Opin Ophthalmol. 2018; 29(6):514-9.

9. Tolou C, Mahieu L, Martin-BlondelG, OlléP, et al. Posterior segment involvement in cat-scratch disease: A case series. J Fr Ophtalmol. 2015; 38(10):974-82.

10. NassifDS. Acute unilateral vision loss in a female adolescent due to Ocular Bartonellosis. Pediatr Emerg Care. 2019; 35(4):e65-6.

11. Fairbanks AM, Starr MR, Chen JJ, Bhatti MT. Treatment Strategies for Neuroretinitis: Current Options and Emerging Therapies. Curr Treat Options Neurol. 2019; 21(8):36.

12. Durá TravéT, Yoldi Petri ME, Lavilla Oiz A, Molins Castiella T. Neurorretinitis asociada a la enfermedad por arañazo de gato. An Pediatr (Barc). 2010; 72(4):290-1.

13. del Valle Welschen DV. Neurorretinitis por Bartonella henselae: presentación de un caso y revisión de la literatura. Rev Mex Oftalmol. 2016; 90(5):235-41. 